\title{
Comparison of Endoscopic Tympanoplasty to Microscopic Tympanoplasty
}

\author{
Nayeon Choi $\cdot$ Yangseop Noh ${ }^{1} \cdot$ Woori Park ${ }^{1}$ Jung Joo Lee ${ }^{1} \cdot$ Sunhyun Yook $\cdot$ Ji Eun Choi $\cdot$ Won-Ho Chung \\ Yang-Sun $\mathrm{Cho}^{1} \cdot$ Sung Hwa Hong ${ }^{1} \cdot||$ Joon Moon $^{1}$ \\ ${ }^{1}$ Department of Otorhinolaryngology-Head and Neck Surgery, Samsung Medical Center, Sungkyunkwan University School of Medicine, Seoul; \\ ${ }^{2}$ Department of Medical Device Management and Research, SAIHST, Sungkyunkwan University, Seoul, Korea
}

Objectives. This study aimed to compare the outcome of endoscopic and microscopic tympanoplasty.

Methods. This was a retrospective comparative study of 73 patients (35 males and 38 females) who underwent type I tympanoplasty at Samsung Medical Center from April to December 2014. The subjects were classified into two groups; endoscopic tympanoplasty (ET, $n=25)$, microscopic tympanoplasty (MT, $n=48$ ). Demographic data, perforation size of tympanic membrane at preoperative state, pure tone audiometric results preoperatively and 3 months postoperatively, operation time, sequential postoperative pain scale (NRS-11), and graft success rate were evaluated.

Results. The perforation size of the tympanic membrane in ET and MT group was $25.3 \% \pm 11.7 \%$ and $20.1 \% \pm 11.9 \%$, respectively $(P=0.074)$. Mean operation time of MT $(88.9 \pm 28.5$ minutes $)$ was longer than that of the ET $(68.2 \pm 22.1$ minutes) with a statistical significance $(P=0.002)$. External auditory canal $(E A C)$ width was shorter in the ET group than in the MT group $(P=0.011)$. However, EAC widening was not necessary in the ET group and was performed in $33.3 \%$ of patients in the MT group. Graft success rate in the ET and MT group were $100 \%$ and $95.8 \%$, respectively; the values were not significantly different $(P=0.304)$. Pre- and postoperative audiometric results including bone and air conduction thresholds and air-bone gap were not significantly different between the groups. In all groups, the postoperative air-bone gap was significantly improved compared to the preoperative air-bone gap. Immediate postoperative pain was similar between the groups. However, pain of 1 day after surgery was significantly less in the ET group.

Conclusion. With endoscopic system, minimal invasive tympanoplasty can be possible with similar graft success rate and less pain.

Keywords. Endoscopy; Tympanoplasty; Minimally Invasive Surgical Procedures; Pain

\section{INTRODUCTION}

Since the introduction of tympanoplasty in the 1950s [1], a variety of graft materials and surgical techniques have been developed and used to close perforations in the tympanic membrane

\footnotetext{
- Received January 10, 2016

Revised February 28, 2016

Accepted March 19, 2016

- Corresponding author: Il Joon Moon

Department of Otorhinolaryngology-Head and Neck Surgery, Samsung

Medical Center, Sungkyunkwan University School of Medicine,

81 Irwon-ro, Gangnam-gu, Seoul 06351, Korea

Tel: +82-2-3410-3579, Fax: +82-2-3410-3879

E-mail: moonij@skku.edu
}

(TM). Temporalis fascia and perichondrium remain the most widely used materials, and successful closure can be achieved in $80 \%$ to $90 \%$ in patients who undergo primary tympanoplasty with a microscopic approach [2]. In some cases, postauricular skin incisions and soft tissue dissections are mandatory during tympanoplasty using a microscope. Conventional microscopic tympanoplasty with a postauricular incision remains the most effective procedure for patients with chronic otitis media, especially in cases of anterior or large TM perforation as well as anterior bony overhang. This conventional procedure results in surgical scar and significant pain to the patient.

Minimally invasive otologic surgery has recently been developed along with endoscopic techniques. Endoscopic ear surgery,

Copyright @ 2017 by Korean Society of Otorhinolaryngology-Head and Neck Surgery

This is an open-access article distributed under the terms of the Creative Commons Attribution Non-Commercial License (http://creativecommons.org/licenses/by-nc/4.0)

which permits unrestricted non-commercial use, distribution, and reproduction in any medium, provided the original work is properly cited. 
first tried in the 1990s [3], has become popular with anatomic and physiologic concepts [4]. Advantages of endoscopic ear surgery compared to the conventional microscopic surgery include avoiding endaural vertical and postauricular incisions, and mastoidectomies in securing the surgical view $[5,6]$. Endoscopically, the typical transcanal approach is possible by elevating a tympanomeatal flap. This avoids other unnecessary incisions and soft tissue dissections. The endoscopic approach also provides better visualization of hidden areas in the middle ear cavity including the anterior and posterior epitympanic spaces, sinus tympani, facial recess, and hypotympanum. Endoscopy-mediated procedures can decrease residual cholesteatomas and recurrences during surgeries for cholesteatoma removal [5,7-9].

The value of endoscopes combined with the conventional microscopic eradication of cholesteatoma has been well established [6-15]. Besides surgeries for cholesteatoma removal, an exclusively endoscopic approach during tympanoplasty has been applied to facilitate minimally invasive surgery $[16,17]$.

However, endoscopic surgery has several disadvantages. Only one-hand surgery is feasible with the endoscopic technique, which is less efficient; in a situation of massive bleeding, the endoscopic view could be stained by blood and continuing the procedure could be difficult. Furthermore, endoscopic instrument could make direct injury and thermal damage by light source $[17,18]$.

There has been lack of reliable data regarding the efficacy and functional outcome of endoscopic tympanoplasty as compared with conventional microscopic tympanoplasty. In this study, we evaluate and compare the results of hearing outcome, postoperative pain assessment, operation time, graft success rate, and surgical complications in patients who underwent endoscopic and conventional microscopic tympanoplasty. The aim is to clarify the clinical benefit of endoscopic tympanoplasty compared to conventional microscopic surgery by an endaural or postauricular approach.

\section{MATERIALS AND METHODS}

This retrospective study was approved by Samsung Medical Center Institutional Review Board (IRB no. 2015-06-095).

\section{H I G G H L L I}

- Endoscopic technique contributes to minimal invasive surgery in tympanoplasty.

- It could achieve better surgical view with minimal incision, reduce postoperative pain.

- Endoscopic tympanoplasty had shorter operation time compared with microscopic surgery.

\section{Subjects}

The study enrolled 73 patients aged 23 to 87 years (mean, $54.0 \pm 12.2$ years) who underwent tympanoplasty type I for chronic otitis media at Samsung Medical Center from April to December 2014. All patients had endoscopic examination, pure tone audiometry, and temporal bone computed tomography as preoperative work-up and had postoperative follow-up with endoscopic examination and pure tone audiometry at 3 months after surgery. Mean follow-up was 6.4 months (range, 3 to 11 months). Patients were classified into two groups according to type of surgery: endoscopic tympanoplasty (ET) and microscopic tympanoplasty (MT). Type of surgery was decided by each surgeon's preference and patient counselling. Either microscopic or endoscopic tympanoplasty were performed by experienced otologists.

\section{Analgesics medication}

All subjects had pain control with acetaminophen $650 \mathrm{mg}$ administered orally three times a day postoperatively for 1 week.

\section{Methods}

Pure tone audiotory tests were performed at preoperatively and 3 months postoperatively. Hearing thresholds including air conduction and bone conduction were measured at 0.5, 1.0, 2.0, and $4.0 \mathrm{kHz}$, and the puretone averages were calculated. TM perforation size was expressed as a percentage of the entire TM area using Image $\mathbf{J}$ software (National Institutes of Health, Bethesda, MD, USA). Detailed operative data were collected including type of operation (ET, MT), operation time, whether external auditory canal (EAC) widening was performed for a better surgical view or not. Width of the narrowest portion of the EAC on the axial image of computed tomography was measured. During the postoperative follow-up, pain scale score was collected immediately after surgery, at 3 hours, and 1 day postoperatively. The graft success rate was also determined. Pain scale was scored using an 11-item, patient-reported numeric rating scale of pain intensity (NRS-11, range 0 to 10). Graft success as well as healing status of the EAC was evaluated at 3 months postoperatively by endoscopic examination. A dry, clean ear canal withoutTM perforation represented graft success.

\section{Surgical technique}

Tympanoplasty type I procedures were performed in all patients. The EAC was widened during the surgery when visualization of the middle ear cavity was difficult due to anterior bony overhang. In the MT group, an Opmi Vario S88 microscope (Carl Zeiss, Oberkochen, Germany) was used, and detailed procedures were almost identical with the ET group. In the MT group, surgery was performed with an endaural incision, based on the Lempert method [19]. The first incision was made along the posterior half of the ear canal. The second vertical incision was made in the incisura to connect the first incision with the area 

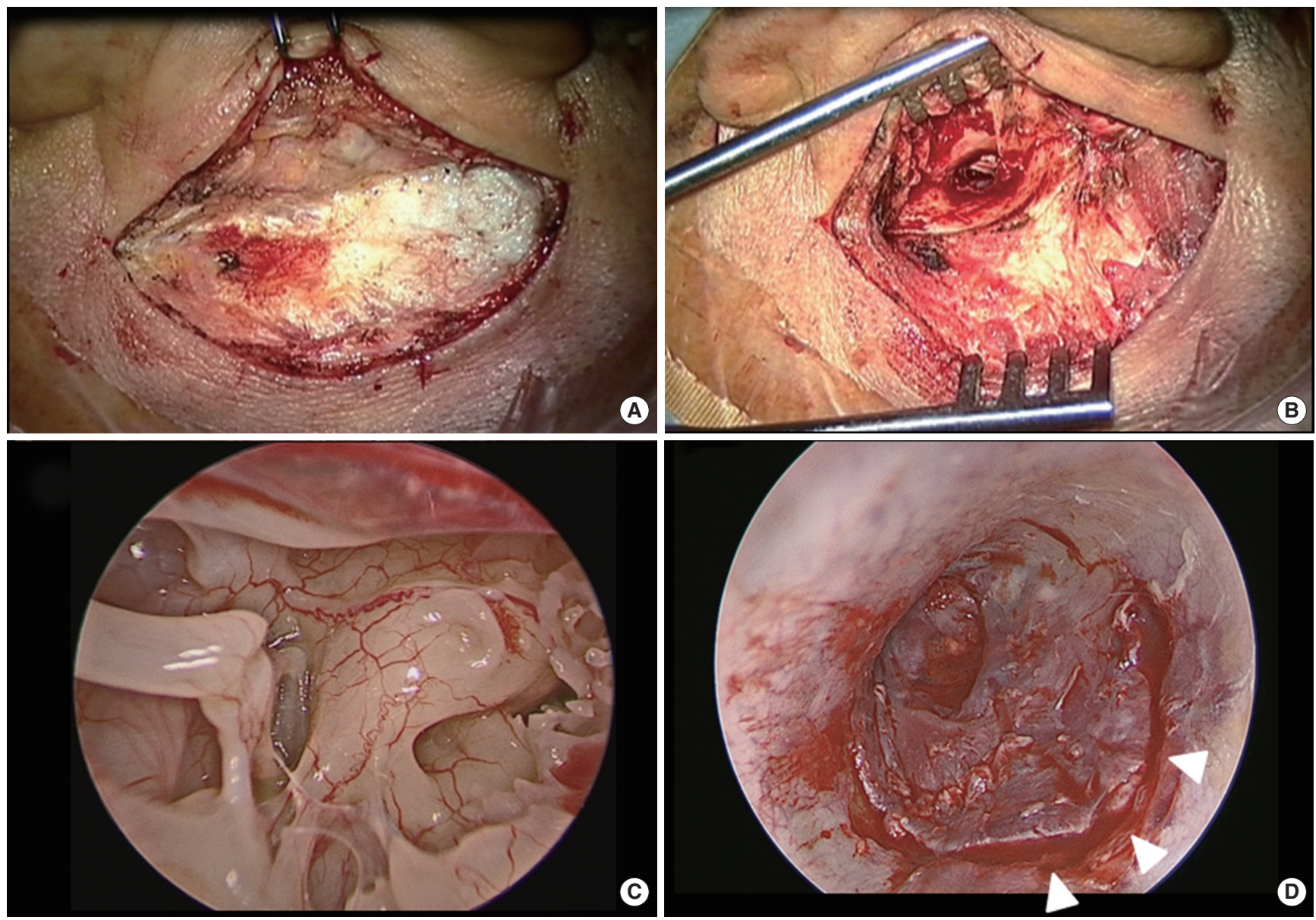

Fig. 1. Details of tympanoplasty. (A) Postauricular incision for microscopic postauricular tympanoplasty. (B) Microscopic view of operation field of tympanoplasty. (C) Endoscopic view of middle ear cavity during endoscopic tympanoplasty. (D) View of transcanal incision (white arrow heads) approach in endoscopic tympanoplasty.

between the tragus and the root of the helix [20]. A postauricular incision was used in cases where the perforation margin was poorly visualized due to narrow external ear canal, anterior bony overhang, or large TM perforation (Fig. 1A, B). In the ET group, an endoscopic system (Karl Storz, Tuttlingen, Germany) and 0- or 30-degree rigid endoscopes (3.0- or 4.0-mm diameter, 11 or $16 \mathrm{~cm}$ long; Karl Storz) were used. In this technique, incisions were performed 5 to $6 \mathrm{~mm}$ lateral to tympanic annulus in the posterior part of the EAC perpendicular to the TM tympanic from the superior and inferior end of the first incision. A tympanomeatal flap was elevated for middle ear cavity visualization, and pathologic processes of the middle ear were removed (Fig. 1C). The middle ear was packed with a Gelfoam (Ferrosan, Soborg, Denmark) and the autologous graft, which was most often harvested from tragal perichondrium and rarely temporalis fascia, was underlaid medial to the TM remnant and the manubrium of the malleus. Finally, the tympanomeatal flap was returned to its original position and the medial aspect of the ear canal was packed with pledgets of Gelfoam (Fig. 1D).

\section{Statistical analyses}

The Wilcoxon rank sum test was used to determine correlation of clinical features between the ET and MT groups. Pre- and postoperative audiometric parameters were compared using paired $t$-tests. Statistical significance was accepted at $P<0.05$. All statistical analyses were performed using SPSS ver. 20.0 (IBM Co., Armonk, NY, USA).

\section{RESULTS}

The demographic data and clinical findings of each group (ET, $\mathrm{n}=25$; MT, $\mathrm{n}=48$ ) are presented in Table 1 . Mean ages were $54.4 \pm 11.7$ years (ET) and 53.7 \pm 12.6 (MT). The ages did not differ significantly. Preoperative audiometric tests including bone and air conduction, and air-bone gap were not significantly different between the two groups $(P=0.174,0.277$, and 0.995 , respectively).

The TM perforation size was larger in ET group $(25.3 \% \pm$ $11.7 \%)$ than MT group $(20.1 \% \pm 11.9 \%)$, without statistical sig- 
Table 1. Comparison between ET and MT groups

\begin{tabular}{|c|c|c|c|c|}
\hline Variable & Overall $(n=73)$ & ET $(n=25)$ & $\mathrm{MT}(\mathrm{n}=48)$ & $P$-value \\
\hline Age (yr) & $54.0 \pm 12.2$ & $54.4 \pm 11.7$ & $53.7 \pm 12.6$ & 0.810 \\
\hline Sex (male:female) & $35: 38$ & 9:16 & $26: 22$ & 0.140 \\
\hline TM perforation (\%) & $21.9 \pm 12.0$ & $25.3 \pm 11.7$ & $20.1 \pm 11.9$ & 0.074 \\
\hline Operation time (min) & $81.8 \pm 28.1$ & $68.2 \pm 22.1$ & $88.9 \pm 28.5$ & $0.002^{*}$ \\
\hline EAC width (mm) & $4.2 \pm 0.6$ & $3.9 \pm 0.7$ & $4.3 \pm 0.6$ & $0.011^{*}$ \\
\hline EAC widening & $16(21.9)$ & 0 & $16(33.3)$ & $0.001^{*}$ \\
\hline Graft success rate & $71(97.3)$ & $25(100)$ & $46(95.8)$ & 0.304 \\
\hline \multicolumn{5}{|l|}{ PTA-preoperative } \\
\hline Bone & $26.6 \pm 16.2$ & $23.9 \pm 16.9$ & $28.0 \pm 15.8$ & 0.174 \\
\hline Air & $45.2 \pm 18.1$ & $42.7 \pm 19.7$ & $46.5 \pm 17.3$ & 0.276 \\
\hline Air-bone gap & $18.7 \pm 7.3$ & $18.9 \pm 7.8$ & $18.6 \pm 7.1$ & 0.995 \\
\hline \multicolumn{5}{|l|}{ Air-bone gap (dB) } \\
\hline Preoperative & $18.7 \pm 7.2$ & $18.9 \pm 1.6$ & $18.6 \pm 1.0$ & 0.877 \\
\hline Postoperative & $11.3 \pm 8.6$ & $9.2 \pm 1.4$ & $12.5 \pm 1.3$ & 0.120 \\
\hline$P$-value & $<0.001^{*}$ & $<0.001^{*}$ & $<0.001^{*}$ & \\
\hline \multicolumn{5}{|l|}{ Bone conduction $(\mathrm{dB})$} \\
\hline Preoperative & $26.6 \pm 16.2$ & $23.9 \pm 16.9$ & $28.0 \pm 15.8$ & 0.174 \\
\hline Postoperative & $27.8 \pm 18.1$ & $29.9 \pm 19.6$ & $29.8 \pm 18.5$ & 0.105 \\
\hline$P$-value & 0.098 & 0.221 & 0.342 & \\
\hline
\end{tabular}

Values are presented as mean \pm standard deviation or number (\%).

ET, endoscopic tympanoplasty; MT, microscopic tympanoplasty; TM, tympanic membrane; EAC, external auditory canal; PTA, pure tone auditory.

${ }^{\star} P<0.05$, statistically significant differences between the groups.

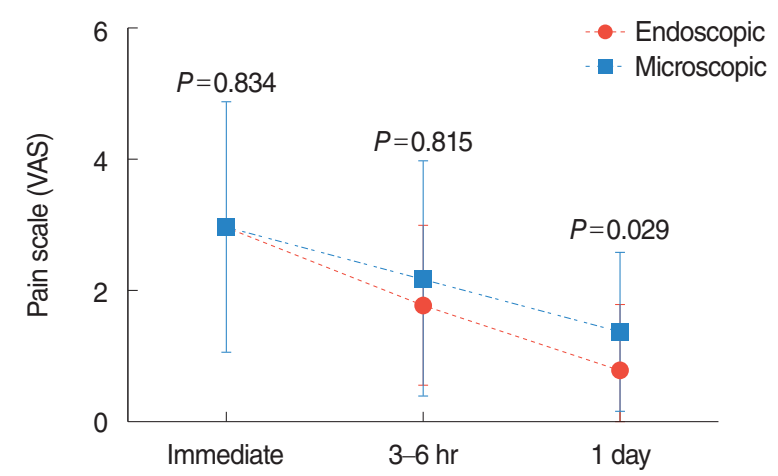

Fig. 2. Comparison of pain scales between the endoscopic and microscopic tympanoplasty group at immediate, 3 to 6 hours, and 1 day after surgery. VAS, visual analogue scale.

nificance $(P=0.074)$. Mean operation time of the MT group (88.9 \pm 28.5 minutes) was significantly longer than the ET group (68.2 \pm 22.1 minutes) $(P=0.002)$. EAC width was shorter in the ET group $(3.9 \pm 0.7 \mathrm{~mm})$ than in the MT group $(4.3 \pm 0.6 \mathrm{~mm})$. However, EAC widening was not necessary in the ET group and was performed in $33.3 \%$ of patients in the MT group. Graft success rate in the ET and MT group was $100 \%$ and $95.8 \%$, respectively, which was not statistically significantly different $(P=$ 0.304).

Pain scale scores were compared between the three groups immediately after surgery, 3 to 6 hours later, and 1 day postoperatively (Fig. 2). Immediately and 3 to 6 hours after surgery, pain scales were not significantly different $(P=0.834$ and 0.815 , respectively). Pain scale score of 1 day after surgery was significantly lower in ET group $(P=0.029)$. The ET group displayed $0.8 \pm 1.0$ of pain score, and MT group was $1.5 \pm 1.3$ at 1 day after surgery.

Preoperative audiometric parameters including bone conduction, air conduction, and air-bone gap were not significantly different between ET and MT group $(P=0.174, P=0.276$, and $P=$ 0.995 , respectively).

Pre- and postoperative air-bone gap was analyzed with paired $t$-test separately in each group. In the ET group, the pre- and postoperative air-bone gap was $18.9 \pm 1.6 \mathrm{~dB}$ and $9.2 \pm 1.4 \mathrm{~dB}$, respectively, which was a significant improvement $(P<0.001)$. The respective values in the MT group $(18.6 \pm 1.0 \mathrm{~dB}$ and $12.5 \pm$ $1.3 \mathrm{~dB})$ also represented a significant $(P<0.001)$.

Bone conduction preoperatively and 3 months postoperatively were compared using the paired $t$-test in each group to evaluate inner ear damage. All groups had no significant difference between pre- and postoperative bone conduction (ET, 23.9 \pm 16.9 vs. $29.9 \pm 19.6 \mathrm{~dB}, P=0.221$; MT, $28.0 \pm 15.8$ vs. $29.8 \pm$ $18.5 \mathrm{~dB}, P=0.342$ ).

In addition, $4.0 \mathrm{kHz}$ bone conduction hearing levels were compared for sensitive evaluation of inner ear damage in each group (Fig. 3). In the ET group, bone conduction hearing level was $25.8 \pm 21.9 \mathrm{~dB}$ preoperatively and $28.2 \pm 20.3 \mathrm{~dB}$ at 3 months postoperatively. There was no significant changes in the ET group $(P=0.200)$. Otherwise, there was significant aggravation of bone conduction in the MT group $(P=0.004)$. Preoperative bone conduction of the MT group was $30.5 \pm 21.0 \mathrm{~dB}$ and postoperative bone conduction was $37.4 \pm 22.8 \mathrm{~dB}$. 


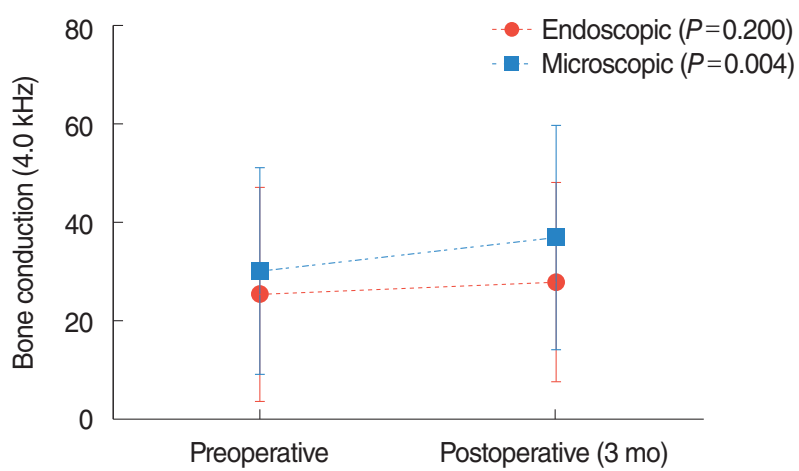

Fig. 3. Comparison of $4.0 \mathrm{kHz}$ bone conduction between the endoscopic and microscopic tympanoplasty group. Pre- and postoperative bone conduction was not significantly changed in the endoscopic tympanoplasty group, but bone conduction hearing threshold was significantly elevated after surgery in the microscopic tympanoplasty group.

\section{DISCUSSION}

Endoscopic ear surgery was first applied to cholesteatoma removal and myringoplasty. Indications are increasing and include middle ear tumor, ossiculoplasty, tympanoplasty, and cochlear implantation [17]. Several meta-analyses and review articles of endoscopic ear surgery support the safety of the approach, with minimal morbidity evident $[15,17,21]$. Since the endoscopic technique was introduced for middle ear surgery, the concept of minimally invasive surgery has developed. This could avoid mastoidectomies, external incisions, and soft tissue dissection in selected cases as compared with the conventional microscopic approach $[9,14]$.

There are still many advantages of microscopic ear surgery. It provides binocular vision along with an excellent magnified surgical view. Using a microscope, two-hand surgery is possible, which is extremely useful to remove blood from the operation field. However, visualization of deep and hidden spaces involving sinus tympani, epitympanum, facial recess, and the attic area are limited with a microscope $[5,9,12,13,17]$. Therefore, microscopic techniques frequently need further soft tissue dissection/ retraction and bony drilling to obtain a better surgical view [17]. An endoscopic system allows surgeons a clear and high-definition surgical view, with less chance of incision and drilling, as the powerful light source is located at the tip of the endoscope, and angled lenses facilitate visualization of hidden recesses [17]. In addition, endoscopic view could provide better chance of education to trainees.

In this study, EAC widening or external incision were not needed in any patient of ET group, although EAC width was significantly narrower in the ET group. Otherwise, one-third of patients who underwent microscopic tympanoplasty had EAC widening by drilling, and three-quarters of patients needed a postauricular incision. Patients who had microscopic postauricu- lar tympanoplasty require postauricular incision care in the form of a compression bandage dressing and had more bloody oozing from the endaural operation site. No externally visible scars were created in the endoscopic group, which is a better cosmetic outcome that requires less postoperative care.

However, endoscopic ear surgery has several disadvantages as compared with microscopic technique. The endoscopic instrument can cause direct injury and thermal damage to the external canal and middle ear $[18,22]$. Furthermore, only one-handed surgery is feasible with the endoscopic technique, which is less efficient; in a situation of massive bleeding, the endoscopic view could be hindered by blood and continuing the procedure could be difficult. The limitation of one-handed surgery would likely be overcome by experience over time. Surgeons who skilled for microscopic surgery could be easily adapted to onehanded surgery with endoscopic system with short duration of learning curve improvement.

Due to heat generation from light source of endoscope, Kozin et al. [22] recommended using submaximal light intensity, and frequent repositioning of the endoscope. In this study, we assumed that there was no inner ear damage by light source, because bone conduction of endoscopic tympanoplasty patients was not significantly changed after surgery, and no patients experienced dizziness or nystagmus during postoperative period.

In spite of the one-handed nature of the endoscopic surgery, the current data indicate that endoscopic tympanoplasty can be successfully performed by an experienced surgeon. Even in patients with large TM perforation or anterior bony overhang, endoscopic tympanoplasty was conducted and graft success rate was comparable to the microscopic group. The endoscopic group had significantly shorter operation time than the microscopic tympanoplasty group. Closure of the postauricular incision in the microscopic postauricular tympanoplasty group might extend the operation time. In addition, the endoscopic technique offered a wide and clean surgical view with minimal canal incision (Fig. 1C, D), which could result in minimal manipulation of soft tissue, bony drilling, and bleeding, and subsequently increased efficacy of surgery as well as reduced pain. Previous study also reported same results that operative time in endoscopic tympanoplasty was significantly shorter than microscopic tympanoplasty [16].

Studies have analyzed hearing outcome after endoscopic ear surgery. One study reported significant hearing improvements in pediatric patients after endoscopic tympanoplasty [16]. Another study demonstrated significant hearing improvements after endoscopic middle ear surgery for cholesteatoma removal [23]. Similarly, significant improvements of air-bone gap after endoscopic tympanoplasty were found presently.

The endoscopic system has been used for second-look procedures or primary resection of middle ear cholesteatoma. Advantages include clear observation of middle ear cavity, low recurrence rate, prevention of a retraction pocket, and preservation of 
ossicles $[9,11,13,24]$. Presently, there were no patients with cholesteatoma, but we achieved a graft success rate of $100 \%$ at 3 months, with no recurrence of otorrhea at 3 months after endoscopic tympanoplasty. Therefore, we suppose that the endoscopic technic could be helpful for eradication of pathologic processes in the middle ear, such as granulation tissue or adhesion, as well as cholesteatoma.

Little is known of postoperative pain after endoscopic ear surgery. Only one study reported that the endoscopic tympanoplasty gave an equal result to microscopic tympanoplasty regarding pain level [16]. In that study, the endoscopic group had significantly lower level of pain than the microscopic groups 1 day after surgery. The reduced pain may reflect the lack of external incision as well as vertical endaural incision up to incisura and EAC bone drilling in the endoscopic technique.

Limitation of this study was that type of surgery (endoscopic or microscopic) was decided by each surgeon's preference and patient counselling which could cause bias. Therefore, we tried to minimize it by following methods. All surgeries were performed by skillful otologic surgeons who had more than 5 years of clinical experience. In addition, preoperative clinical data such as age, sex, hearing and size of TM perforation were compared, and they were not significantly different between two groups. In future, further well-designed randomized controlled trial is needed to overcome the bias.

In summary, the endoscopic technique had shorter operation time and has reduced pain level compared with microscopic tympanoplasty. Regardless of the perforation size, there was no need for external incision or EAC drilling in the ET group, which may lead to better patient satisfaction.

For tympanoplasty, the endoscopic technique contributes to minimal invasive surgery with transcanal incision only. It could minimize additional incision, EAC widening for better surgical view, and especially postoperative pain.

\section{CONFLICT OF INTEREST}

No potential conflict of interest relevant to this article was reported.

\section{REFERENCES}

1. Zollner F. The principles of plastic surgery of the sound-conducting apparatus. J Laryngol Otol. 1955 Oct;69(10):637-52.

2. Dornhoffer JL. Hearing results with cartilage tympanoplasty. Laryngoscope. 1997 Aug;107(8):1094-9.

3. Thomassin JM, Duchon-Doris JM, Emram B, Rud C, Conciatori J, Vilcoq P. Endoscopic ear surgery: initial evaluation. Ann Otolaryngol Chir Cervicofac. 1990;107(8):564-70.

4. Marchioni D, Alicandri-Ciufelli M, Piccinini A, Genovese E, Presutti
L. Inferior retrotympanum revisited: an endoscopic anatomic study. Laryngoscope. 2010 Sep;120(9):1880-6.

5. Ayache S, Tramier B, Strunski V. Otoendoscopy in cholesteatoma surgery of the middle ear: what benefits can be expected? Otol Neurotol. 2008 Dec;29(8):1085-90.

6. Migirov L, Shapira Y, Horowitz Z, Wolf M. Exclusive endoscopic ear surgery for acquired cholesteatoma: preliminary results. Otol Neurotol. 2011Apr;32(3):433-6.

7. Thomassin JM, Korchia D, Doris JM. Endoscopic-guided otosurgery in the prevention of residual cholesteatomas. Laryngoscope. 1993 Aug;103(8):939-43.

8. Good GM, Isaacson G. Otoendoscopy for improved pediatric cholesteatoma removal. Ann Otol Rhinol Laryngol. 1999 Sep;108(9): 893-6.

9. Presutti L, Marchioni D, Mattioli F, Villari D, Alicandri-Ciufelli M. Endoscopic management of acquired cholesteatoma: our experience. J Otolaryngol Head Neck Surg. 2008 Aug;37(4):481-7.

10. Badr-el-Dine M. Value of ear endoscopy in cholesteatoma surgery. Otol Neurotol. 2002 Sep;23(5):631-5.

11. Tarabichi M. Endoscopic management of limited attic cholesteatoma. Laryngoscope. 2004 Jul;114(7):1157-62.

12. Marchioni D, Mattioli F, Alicandri-Ciufelli M, Presutti L. Endoscopic approach to tensor fold in patients with attic cholesteatoma. Acta Otolaryngol. 2009 Sep;129(9):946-54.

13. Tarabichi M. Transcanal endoscopic management of cholesteatoma. Otol Neurotol. 2010 Jun;31(4):580-8.

14. Marchioni D, Villari D, Alicandri-Ciufelli M, Piccinini A, Presutti L. Endoscopic open technique in patients with middle ear cholesteatoma. Eur Arch Otorhinolaryngol. 2011 Nov;268(11):1557-63.

15. Presutti L, Gioacchini FM, Alicandri-Ciufelli M, Villari D, Marchioni D. Results of endoscopic middle ear surgery for cholesteatoma treatment: a systematic review. Acta Otorhinolaryngol Ital. 2014 Jun; 34(3):153-7.

16. Dundar R, Kulduk E, Soy FK, Aslan M, Hanci D, Muluk NB, et al. Endoscopic versus microscopic approach to type 1 tympanoplasty in children. Int J Pediatr Otorhinolaryngol. 2014 Jul;78(7):1084-9.

17. Kozin ED, Gulati S, Kaplan AB, Lehmann AE, Remenschneider AK, Landegger LD, et al. Systematic review of outcomes following observational and operative endoscopic middle ear surgery. Laryngoscope. 2015 May;125(5):1205-14.

18. Bottrill I, Perrault DF Jr, Poe D. In vitro and in vivo determination of the thermal effect of middle ear endoscopy. Laryngoscope. 1996 Feb;106(2 Pt 1):213-6.

19. Lempert J. Endaural, antauricular surgical approach to the temporal bone principles involved in this new approach: summary report of 1,780 cases. Arch Otolaryngol. 1938 May;27(5):555-87.

20. Gulya AJ, Minor LB, Poe DS. Glasscock-Shambaugh's surgery of the ear. 6th ed. Shelton, CT: People's Medical Publishing House-USA; 2010.

21. Marchioni D,Alicandri-Ciufelli M, Gioacchini FM, Bonali M, Presutti L. Transcanal endoscopic treatment of benign middle ear neoplasms. Eur Arch Otorhinolaryngol. 2013 Nov;270(12):2997-3004.

22. Kozin ED, Lehmann A, Carter M, Hight E, Cohen M, Nakajima HH, et al. Thermal effects of endoscopy in a human temporal bone model: implications for endoscopic ear surgery. Laryngoscope. 2014 Aug; 124(8):E332-9.

23. Hanna BM, Kivekas I, Wu YH, Guo LJ, Lin H, Guidi J, et al. Minimally invasive functional approach for cholesteatoma surgery. Laryngoscope. 2014 Oct;124(10):2386-92.

24. Barakate M, Bottrill I. Combined approach tympanoplasty for cholesteatoma: impact of middle-ear endoscopy. J Laryngol Otol. 2008 Feb;122(2):120-4. 